\title{
Image Super-Resolution Reconstruction based on Multi-Groups of Coupled Dictionary and Alternative Learning
}

\author{
Sun Guangling \\ School of Communication and \\ Information Engineering \\ Shanghai University \\ China \\ 200072
}

\author{
Li Guoqing \\ School of Communication and \\ Information Engineering \\ Shanghai University \\ China \\ 200072
}

\author{
Jiang Xiaoqing \\ School of Communication and \\ Information Engineering \\ Shanghai University \\ China \\ 200072
}

\begin{abstract}
A novel image super-resolution reconstruction framework based on multi-groups of coupled dictionary and alternative learning is investigated in this paper. In dictionary learning phase, each image of a training image set is taken as high resolution image (HRI), the reduced and re-enlarged result of HRI by interpolation is taken as low resolution image (LRI), and the difference between them is residual image. To obtain the mapping between residual and LRI, coupled dictionaries are learned from joint data composed of residual image patch and LRI patch features. Considering that distinguished texture and structural characteristics reflected in image patches and dictionary learning efficiency as well, multi-groups of coupled dictionary and alternative learning scheme are proposed. In reconstruction phase, LRI is obtained first. Then sparse representations and corresponding errors are calculated for each patch of the LRI by using low resolution component of each group of coupled dictionary. The residual component of coupled dictionary with minimum errors is applied to reconstruct the corresponding residual image patch. All such reconstructed residual patches compose a residual image. Finally, the residual image and the LRI are fused to produce an expected HRI. An experimental study is performed to establish that the proposed approach improves the super-resolution reconstruction quality.
\end{abstract}

\section{Keywords}

super-resolution, sparse representation, multi-dictionary, alternative learning, principal subspace, orthogonal Gaussian mixture model

\section{INTRODUCTION}

Image Super-Resolution reconstruction (SR), as one of the most valuable research topics, its aims is to get beyond the resolution of imaging sensors or obtain a higher resolution image. It has a variety of useful applications. Usually, a high-resolution image is reconstructed from one or multiple low-resolution images. If multiple low-resolution images are available, they are registered at sub-pixel accuracy and a higher resolution image is generated by reconstruction or interpolation. However, with the expected scale of super resolution is enlarged, the performance of SR will decay even if the number of low-resolution image is increased. In a word, when a large SR scale is required or low-resolution images are insufficient, SR only using low-resolution images will easily fail.

To break the limitation, numerous learning based schemes have been proposed in recent years. Relying on the learned prior knowledge, the SR reconstruction performed well whereas the low-resolution image number is reduced significantly even only a single low-resolution image is available. In fact, learning based schemes have been well popular in super-resolution and other image processing tasks. The crucial point of it is to learn a mapping between low frequency and high frequency and the learned mapping is adopted to obtain the high-resolution image given a low-resolution image. Freeman proposed the example-based method [1]. As one of the earliest learning-based SR reconstruction approaches, it modeled the spatial relations of image patches using Markov-network to obtain the transfer 
probability matrix between high resolution image patches or high and low resolution image patches. Rich high frequency information thereafter was estimated relying on the learned matrix. However, the training images must be selected carefully. In addition, the method is sensitive to noise and the learning efficiency is relative low. Chang proposed neighbor embedding [2]. It is assumed that corresponding high and low resolution patches have same local geometrical manifold. First, low and high dimensional manifolds were learned. Then k-nearest neighbor representations of a low resolution patch were searched in low dimensional manifold, and the weighted representations and high dimensional manifold are used to reconstruct the high resolution patch. But for low and high resolution patches, the neighbor relations would not always be preserved. To overcome the limitation, Chan improved neighbor embedding from several respects, such as residual neighbor embedding [3], edge detection and feature selection [4].Compared to the example-based method, neighbor embedding required less training samples and is less sensitive to noise. Ni proposed a SR method based on Super Vector Regression (SVR) [5]. By integrating additional constraints, they transformed the kernel learning from a positive semi-definite programming problem into a quadratic linear programming problem. In this method, samples were selected automatically and training set was not large. Moreover, it was effective in both spatial and DCT domain. The robustness to noise present in image was an advantage of this method. Nevertheless, the high computation complexity of non-linear SVR prohibits SR task with large-scale images.

Recently, sparse representation and dictionary learning have become one of the most important tools to address a wide range of image processing problems including super resolution. Yang calculated sparse representations for raw image patches [6]. In learning phase, they sampled a large amount of pairs of image patches randomly from an outer image set. Joint features composed of low and high features extracted from high and low resolution patches were directly used as a pair of redundant dictionary. In reconstruction phase, features were extracted from low resolution image and its sparse representations were applied with high part of the redundant dictionary to recover a high resolution image patch. Since the sparse coding was determined accordingly through algorithm, it is not necessary to set the element number in advance whereas the neighbor size must be set in neighbor embedding. Compared with directly using image patched as a dictionary, Yang improved his own method by learning a dictionary to generate a more compact representation of patch pairs, reducing the calculation complexity significantly [7]. Yang's work, which first applied sparse representation in image super resolution, is a pioneer research in related field. However, because of the large variation of data, a single dictionary is not capable of representing data sparsely with a high precision. In addition, Lagrange Dual and Lasso of which efficiency were not satisfied were adopted to learn the dictionary. Again in framework of sparse representation, Zeyde proposed to train dictionary from the low resolution input image itself [8]. Unlike the strategy of joint dictionary learning proposed by Yang, Zeyde first learned the low resolution dictionary using K-SVD algorithm [9] and then obtained the high resolution dictionary with MOD algorithm [10]. Although both learning efficiency and reconstruction quality were improved, the method still learned a single dictionary and a dictionary must be relearned for each input low resolution image which undoubtedly dropped the reconstruction efficiency. Moreover, the heavy computation burden of K-SVD is also an annoying problem. Yang proposed SVR integrated with sparse representations [11]. The support vector repressors and image patch category were two crucial factors of this work and the computation complexity of it is substantially reduced than that of existing SVR based methods.

To overcome the previous drawbacks in sparse representation framework, we propose multi-groups of coupled dictionary and its alternative learning algorithm. Our first contribution is the multi-groups of dictionaries. In principal subspace of low resolution features, Orthogonal Gaussian Mixture Models (OGMM) [12] is used to classify the low resolution image patch automatically according to their features. In a sequel, a coupled dictionary is learned by using low and high resolution patches in respective patch category space so that multiple coupled dictionaries are produced. During reconstruction, the sparse representations and reconstruction error of low resolution patch corresponding to low part of each coupled dictionary are calculated. Next, the high resolution patch is recovered using the calculated sparse representations and high part of the coupled dictionary that has least reconstruction error. In a brief, high frequency is complemented adaptively according to the local visual content. Another contribution is 
the alternative learning of coupled dictionary. Alternative learning use low and high resolution data alternatively and MOD algorithm to guarantee low and high resolution dictionary are synchronized. At the same time, the learning efficiency is also improved greatly.

\section{FRAMEWORK AND ALGORITHM OF MULTI-DICTIONARY SCHEME}

Statistics learning theory [13] shows that a dramatic augment of training sample amount will definitely enhance the generalization of dictionary. However, the variation of data will also enlarge with many types of textures and edges appearing. Clearly, a single dictionary is not sufficient to represent data both sparsely and accurately. In other words, single dictionary is under-fitting. Hence, data is categorized into multiple groups based on its visual characteristics. After that, dictionary is learned in respective data group. This strategy will effectively reduce the bias of reconstruction error with sparse representation and considerably boost the overall performance of dictionary.

The framework consists of two phases: learning phase and reconstruction phase, which have been shown in Fig 1 and Fig 2 respectively. In learning stage, we randomly sample a large number of image patches from the low resolution images and residual images at corresponding positions, which residual image is difference between high resolution and low resolution image. Then, features are extracted from the residual and low resolution image jointly written as $\left\{\mathrm{p}_{r}, \mathrm{p}_{l}\right\} . \mathrm{p}_{l}$ is used and clustered by solving OGMM, generating multi-groups of data. Upon each group of data, a coupled dictionary composed of residual part $\mathrm{D}_{r}$ and low-resolution part $\mathrm{D}_{l}$ is learned with alternative learning. In reconstruction phase, low resolution patch features are extracted first. Next, their sparse representations and corresponding reconstruction errors using $\mathrm{D}_{l}$ of each coupled dictionary is computed. Depending on the sparse representations and $\mathrm{D}_{r}$ of coupled dictionary corresponding to the least errors, the residual patch is estimated. Last, low resolution patch and residual patch are merged to generate a high resolution patch. The crucial goal of multiple dictionaries is to choose the most appropriate dictionary to reconstruct so as to improve the quality of reconstructed image. For alternative learning, residual data and low resolution data are learned alternatively with MOD algorithm. Consequently, both high learning efficiency and synchronization between two parts of coupled dictionary are obtained.

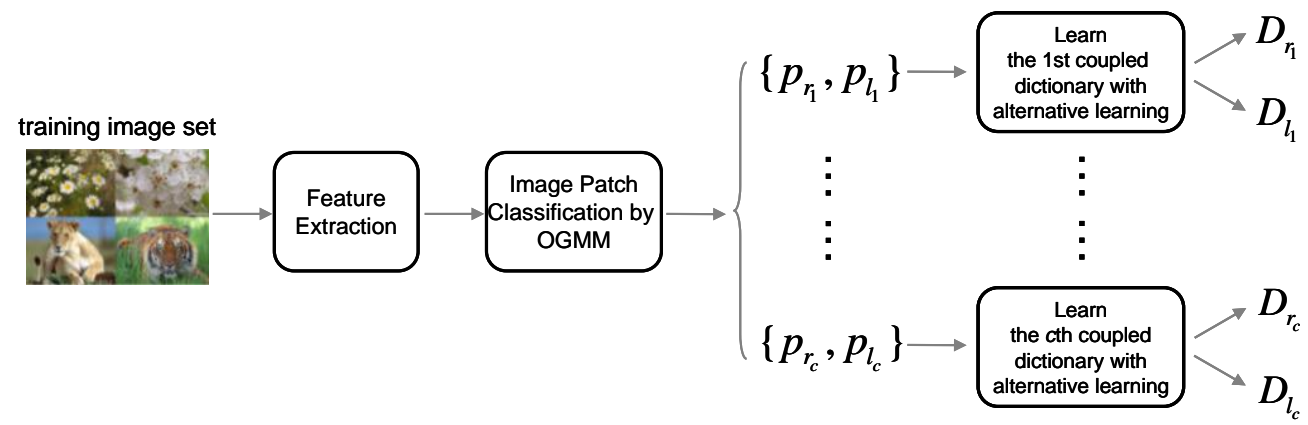

Fig 1: Overview of learning phase

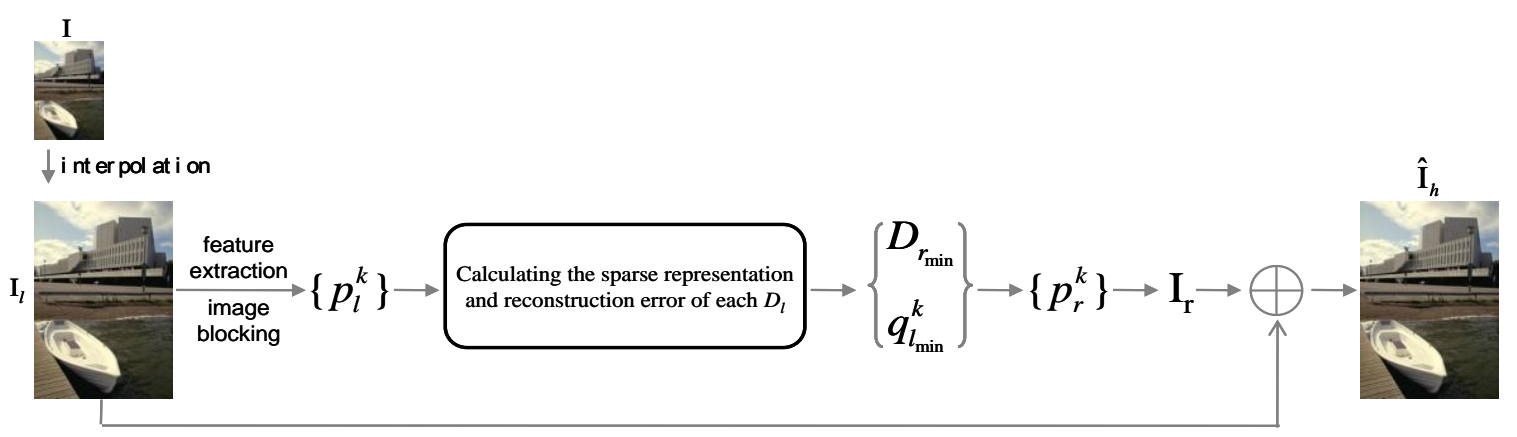

Fig 2: Overview of reconstruction phase 


\subsection{Learning phase}

\subsubsection{Training samples generation}

Similar to other learning based methods, low and high resolution training images are produced artificially as follows:

$$
y_{l}^{j}=\mathrm{ASH} \cdot y_{h}^{j}+\mathrm{A} \cdot v
$$

Where $y_{h}^{j}$ is $j$ th original image regarded as a high resolution image, $\mathrm{H}$ denotes a blurring operator, $\mathrm{S}$ denotes a down sampling operator, A denotes an interpolation process, $v$ is an additive Gaussian white noise. After a series of operations, $j$ th low resolution image $y_{l}^{j}$ is generated accordingly.

\subsubsection{Image Patch Classification based on OGMM in Principal Subspace}

\section{- Features for image patch classification}

The edge features of low resolution patch are chosen for image patch classification. Each patch is filtered by 4 high-pass filters $f_{r}, r=1,2,3,4$, which are differential filters with first and second order, horizontal and vertical directions respectively. From the four filtered images, four corresponding patches which are size of $\sqrt{n} \times \sqrt{n}$ and position at $k \in \Omega$, are extracted and concatenated into a complete feature vector $\tilde{p}_{l}^{k}$. Assume $n=81$, the dimension of $\tilde{p}_{l}^{k}$ is $4 * 81=324$. Obviously, if $\tilde{p}_{l}^{k}$ is directly adopted to classify patches or coupled dictionary learning and sparse coding, the computation complexity will rather high. Hence, the original feature space is first transformed into an orthogonal space with K-L transform; then the principal subspace is selected as the space in which image patch classification is accomplished.

\section{- OGMM in principal subspace and image patch classification}

In brief, OGMM models the mixtures of Gaussian distribution of the data in orthogonal space. Due to energy concentration along the principal axis that features distribute, OGMM achieves a more effective data distribution. Compared with full-variance Gaussian mixture model, OGMM substantially decreases the number of free parameters and thus reduces the computational and storage cost of the related calculations. Its formulation is defined as follows:

$$
p\left(x \mid w_{c}, \varphi_{c}, \Sigma_{c}, c=1, \ldots, C\right)=\sum_{c=1}^{C} w_{c} O_{c}\left(\Omega^{T} x \mid \varphi_{c}, \Sigma_{c}\right)
$$

$$
\begin{aligned}
& O_{c}\left(\Omega^{T} x \mid \varphi_{c}, \sum_{c}\right) \\
& =\frac{1}{(2 \pi)^{d / 2}\left|\sum_{c}\right|^{1 / 2}} \exp \left[-\frac{1}{2}\left(\Omega^{T} x-\varphi_{c}\right)^{T} \sum_{c}^{-1}\left(\Omega^{T} x-\varphi_{c}\right)\right] \\
& \quad, \sum_{c=1}^{C} w_{c}=1
\end{aligned}
$$

Where $x$ denotes the original feature vector and $d$ is the dimension of it; $C$ denotes the number of Gaussian mixture components, $w_{c}, \varphi_{c}$ and $\sum_{c}$ denotes the weights, mean vector and diagonal covariance matrix of Gaussian components respectively, $\Omega$ is K-L orthogonal transform matrix. The parameters of OGMM can be resolved by Expectation Maximization (EM) algorithms. For the problem in this paper, $x$ is $\tilde{p}_{l}^{k}$; since $\tilde{p}_{l}^{k}$ must be compressed, the dimensionality of $\Omega$ named principal subspace is in fact $324 \times n_{l}$. The value of $n_{l}$ is determined by required preserved energy and super resolution scale. For instance in our work, the required preserved energy is $99.9 \%$ and super resolution scale is 3 , $n_{l}$ is about 48. EM algorithm is run to obtain $\hat{\varphi}_{c}$ and $\hat{\Sigma}_{c}, c=1,2, \ldots, C$, which are likelihood parameters of each image patch category. For each input image patch feature $\tilde{p}_{l}^{k}$, the likelihood $p\left(\Omega^{T} \tilde{p}_{l}^{k} \mid \hat{\varphi}_{c}, \hat{\Sigma}_{c}\right)$ of each category is calculated by equation (3) and the category corresponding to the maximum likelihood is the patch belonging to.

$$
\begin{aligned}
& p\left(\Omega^{T} \tilde{p}_{l}^{k} \mid \hat{\varphi}_{c}, \hat{\Sigma}_{c}\right) \\
& =\frac{1}{(2 \pi)^{n_{l} / 2}\left|\hat{\Sigma}_{c}\right|^{1 / 2}} \exp \left[-\frac{1}{2}\left(\Omega^{T} \tilde{p}_{l}^{k}-\hat{\varphi}_{c}\right)^{T} \hat{\Sigma}_{c}^{-1}\left(\Omega^{T} \tilde{p}_{l}^{k}-\hat{\varphi}_{c}\right)\right]
\end{aligned}
$$

\subsubsection{Features for coupled dictionary learning and alternative learning}

Upon the classification results, coupled dictionary is learned with alternative learning from the residual and low resolution data of each category.

\section{- Features for coupled dictionary learning}

A coupled dictionary contains two parts: residual part and low resolution part. The former is learned from residual data, and the latter is learned from low resolution data which is compressed and projected adaptively upon its own subspace $\Omega_{c}, c=1,2, \cdots C$. Certainly, the two parts of coupled dictionary must be synchronized naming 'coupled'. It is worth mentioning that why residual data other than high resolution data is utilized. 'Residual' more emphasizes the 
differences among subareas that contain more high frequency components such as edges and texture patches. It is reasonable that adopting residual data is capable of recovering more high frequency lost by low resolution image.

\section{- Alternative Learning}

Given residual data $\mathrm{P}_{r}$ and low resolution data $\mathrm{P}_{l}$, a coupled dictionary is learned by running the following alternative learning.

\section{Step 1:}

The dictionary atom number is preset. The preset number of data from $\mathrm{P}_{r}$ or $\mathrm{P}_{l}$ are selected randomly as an initialization of dictionary denoted as $\mathrm{D}_{a}^{(0)}$. In following symbols, if $\mathrm{P}_{b}$ and $\mathrm{Q}_{a}^{(n)}$ refer to low resolution data and dictionary, $\mathrm{P}_{b}$ and $\mathrm{D}_{b}$ refer to high resolution data and dictionary and vice versa. Let $n$ denotes iteration number and its initial value is 1 .

\section{Step 2:}

- Normalize $\mathrm{D}_{a}^{(n-1)}$.

- Utilize $\mathrm{P}_{a}$ and normalized $\mathrm{D}_{a}^{(n-1)}$, the sparse representation $\mathrm{Q}_{a}^{(n)}$ is searched by orthogonal matching pursuit (OMP) algorithm [14].

$$
\begin{aligned}
& \mathrm{E}(\mathrm{Q})=\min \left\|\mathrm{P}_{a}-\mathrm{D}_{a}^{(n-1)} \mathrm{Q}\right\|_{F}^{2}, \text { s.t. }\left\|q^{k}\right\|_{0} \leq L, \forall k \\
& \left\{\mathrm{Q}_{a}^{(n)}\right\}=\arg \min \mathrm{E}(\mathrm{Q})
\end{aligned}
$$

Where $L$ denotes the sparsity. The reason that the dictionary has to be normalized is OMP algorithm requires Euclidean norm of atom to be 1 .

\section{Step 3:}

- Utilize $\mathrm{P}_{b}$ and pseudo-inverse of $\mathrm{Q}_{a}^{(n)}, \mathrm{D}_{b}^{(n)}$ is obtained by MOD algorithm:

$$
\begin{gathered}
\mathrm{D}_{b}^{(n)}=\mathrm{P}_{b}\left(\mathrm{Q}_{a}^{(n)}\right)^{+}=\mathrm{P}_{b}\left(\mathrm{Q}_{a}^{(n)}\right)^{T}\left[\left(\mathrm{Q}_{a}^{(n)}\right)\left(\mathrm{Q}_{a}^{(n)}\right)^{T}\right]^{-1} \\
\mathrm{D}_{r}=\mathrm{D}_{b}^{(n)} \text { Or } \quad \mathrm{D}_{l}=\mathrm{D}_{b}^{(n)}
\end{gathered}
$$

Where $\mathrm{Q}_{a}^{(n)}$ is a full-row rank matrix.

- Exchange data and dictionary letting $\mathrm{D}_{a}^{(n)}=\mathrm{D}_{b}^{(n)}$ and $\mathrm{P}_{a}=\mathrm{P}_{b}$. In addition, $n=n+1$.

\section{Step 4:}

Execute step2 and step 3 alternately until the preset iteration number is reached. Finally, $\hat{\mathrm{D}}_{l}$ and $\mathrm{D}_{l}$ should be normalized to be $\hat{\mathrm{D}}_{r}$ and $\hat{\mathrm{D}}_{l}$.

In equation (5), sparse representations of residual data and low resolution data are used to get low resolution dictionary or sparse representations of low resolution data and residual data are used to get residual dictionary. The two computations are executed alternatively for the purpose of synchronizing the two dictionaries. That is to say, relying on identical sparse representations, such a coupled dictionary could be employed to approximate its own data with minimum or near minimum representation errors. Step 2 and step 3 are performed iteratively to further optimize the coupled dictionary. In alternative learning, initial sparse representation $\mathrm{Q}_{a}^{(1)}$ is also an extreme important, for it will affect the performance of dictionary. Because the features of low resolution data rather than residual data are compressed, residual data is selected to initialize the dictionary $\mathrm{D}_{r}^{(0)}$ and $\mathrm{Q}_{r}^{(1)}$ is obtained correspondingly.

In addition, during reconstruction, the sparse representations using $\hat{\mathrm{D}}$, need to be compensated with atom length of unnormalized dictionary $\tilde{\mathrm{D}}_{l}=\left(\tilde{d}_{1}, \cdots \tilde{d}_{m}\right)$. The normalized version of it is $\hat{\mathrm{D}}_{l}=\left(\hat{d}_{1}, \cdots \hat{d}_{m}\right), \quad\left\|\hat{d}_{1}\right\|=\cdots=\left\|\hat{d}_{m}\right\|=1$. Thus,

$$
\begin{aligned}
\left\|\mathrm{P}_{l}-\tilde{\mathrm{D}}_{l} \hat{\mathrm{Q}}\right\|_{F}^{2} & =\left\|\mathrm{P}_{l}-\left(\hat{d}_{1} \cdot\left\|\tilde{d}_{1}\right\|, \cdots, \hat{d}_{m} \cdot\left\|\tilde{d}_{m}\right\|\right) \hat{\mathrm{Q}}\right\|_{F}^{2} \\
& =\left\|\mathrm{P}_{l}-\left(\hat{d}_{1}, \cdots, \hat{d}_{m}\right)\left(\left\|\tilde{d}_{1}\right\|, \cdots,\left\|\tilde{d}_{m}\right\|\right) \hat{\mathrm{Q}}\right\|_{F}^{2} \\
& =\left\|\mathrm{P}_{l}-\left(\hat{d}_{1}, \cdots, \hat{d}_{m}\right) \hat{\mathrm{Q}}^{\prime}\right\|_{F}^{2} \\
\hat{\mathrm{Q}} & =\hat{\mathrm{Q}}^{\prime} /\left(\left\|\tilde{d}_{1}\right\|, \cdots,\left\|\tilde{d}_{m}\right\|\right), \quad i=1,2, \ldots, m
\end{aligned}
$$

Where $m$ denotes the atom number, \|\|$\|$ denotes the length of each atom, each element of each column of $\hat{Q}^{\prime}$ is divided by $\left\|\tilde{d}_{i}\right\|, i=1,2, \cdots m$. In other words, $\hat{\mathrm{Q}}^{\prime}$ is obtained using $\hat{\mathrm{D}}_{l}$ but $\hat{\mathrm{Q}}$ is required to estimate the residual data. Their relations are connected through equation (7).

\section{- Comparisons to other coupled dictionary learning approaches}

Other coupled dictionary learning approaches such as one proposed by Yang [7]. He combined the features of low and high resolution patch to learn the couple dictionary with 
Lagrange Dual and Lasso algorithm. After the dictionary is learned, it is separated into high and low resolution parts. The dimension of joint feature is high, thus the computation efficiency is relatively low. Zeyde [8] first learned the low resolution dictionary using K-SVD algorithm, and then learned the high resolution dictionary by MOD algorithm. Apparently, the learning efficiency has been improved at cost of declining reconstruction quality. We propose to learn the coupled dictionary in alternative manner making them to be synchronized. The convergence speed of alternative learning is rapid such that only a few iterations are enough to get a good result. Compared with Yang's and Zeyde's methods, the proposed alternative learning has much higher efficiency and excellent reconstruction quality has been demonstrated in the experimental results.

\subsection{Reconstruction Phase}

The reconstruction phase composes four steps described as follows:

1) Input image $I$ is enlarged to be low resolution image $I_{l}$ with scale $s$ and bicubic interpolation algorithm.

2) $\mathrm{I}_{l}$ is filtered with the same four high-pass filters as in learning phase to produce a set of filtered images $\left\{f_{r} * I_{l}\right\}_{r=1,2,3,4}$. Four $\sqrt{n} \times \sqrt{n}$ patches are extracted from the four filtered images in corresponding positions denoted as $k \in \Omega$. The four patches are merged into a feature vector $\tilde{p}_{l}^{k}, k=1,2, \ldots, N$, where $N$ refers to the total number of patches in $\mathrm{I}_{l}$.

3.1) $\tilde{p}_{l}^{k}$ is projected into each subspace $\Omega_{c}$ of each patch category to generate compressed feature vector $p_{l_{c}}^{k}, c=1,2, \ldots, C$.The sparse representation of it denoted as $\mathrm{q}_{l_{c}}^{k}$ is computed by $\hat{\mathrm{D}}_{l_{c}}$ of each coupled dictionary and OMP algorithm.

3.2) Representation error $\mathrm{E}_{c}=\left\|p_{l_{c}}^{k}-\hat{\mathrm{D}}_{l_{c}} \mathrm{q}_{l_{c}}^{k}\right\|_{2}^{2}$ is computed for each category. Let $\mathrm{q}_{l_{\min }^{k}}^{k}$ and $\hat{\mathrm{D}}_{r_{\min }}$ denote the sparse representations and residual part of couple dictionary that have gained the minimum representation errors. A residual image patch $\hat{p}_{r}^{k}$ corresponding to $\tilde{p}_{l}^{k}$ is thus stimated with $\hat{\mathrm{D}}_{r_{\min }}$ and $\mathrm{q}_{l_{\min }}^{k}$. Notice that $\mathrm{q}_{l_{\min }}^{k}$ must be compensated as done in equation (7).

4) Position $\hat{p}_{r}^{k}$ in their original orders producing residual image $\mathrm{I}_{r}$ and overlapping exist between patches. The pixel value of overlapped positions is an average of all values at those positions. The sum of $\mathrm{I}_{l}$ and $\mathrm{I}_{r}$ are adopted as the reconstructed high resolution image $\hat{\mathrm{I}}_{h}$.

\section{EXPERIMENTAL RESULTS AND ANALYSIS}

The same training image set as the Yang's [7] is selected as our training set. Altogether 80,000 patch pairs are sampled randomly from the training set. To cope with color image, RGB space is transformed into $\mathrm{YCbCr}$ space since $\mathrm{Cb}$ and $\mathrm{Cr}$ channels contain much less high frequency. It means that only $\mathrm{Y}$ channel is super-resolved and $\mathrm{Cb}-\mathrm{Cr}$ channels are enlarged with simple bicubic interpolation algorithms.

\subsection{Objective Evaluation}

Four grayscale images and three color images in Kodak's true color image suite are chosen as test images. The up sample scale is 3 , and the objective evaluation criterion is peak signal-to-noise ratio (PSNR, in $\mathrm{dB}$ ).

The compared methods are bicubic interpolation, Yang's[7], Zeyde's[8], single coupled dictionary with alternative learning(SD+AL), multi-groups of coupled dictionary with GMM and alternative learning (MD+OGMM+AL) respectively shown in Table. 1 . In the four dictionary-based methods, the number of atom of each dictionary is all set 1024.

The boldface in table 1 shows that the method $\mathrm{MD}+\mathrm{OGMM}+\mathrm{AL}$ has obtained the highest PSNR on average level.

\subsection{Subjective Evaluation}

To compare super resolution reconstruction result from subjective evaluation given by human visual system, we select two color images named "kitchen" and "boat" with up sample scale 3. The super resolution results of several regions illustrated by red rectangles are emphasized in Fig 4, Fig 5, Fig 6 and Fig 7 respectively. All the demonstrated regions are their actual sizes.

From Fig 4 and Fig 5, it can be observed that Yang's method 
has good sharpness around salient edges but pseudo-edges and noise in local areas brought by reconstruction is also obvious. The same artifacts have happened in Zeyde's method. For (SD+AL) method, the reconstruction quality has been improved to certain degree. From the comparison of Fig 6 and Fig 7, (MD+OGMM+AL) is advantage over ( $\mathrm{SD}+\mathrm{AL})$ because the edges reconstructed by the former look more natural and the sharpness is still preserved. Compared with other methods, the super resolution image reconstructed by the proposed multi-groups of dictionary has an appropriate visual quality since its whole noise is the lowest and the reconstructed edges demonstrate a good balance between noise and sharpness. To sum up, a best reconstruction has been obtained by the proposed approach.

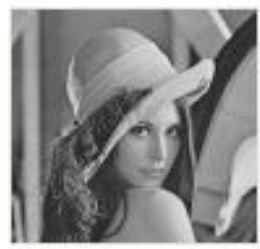

Lena

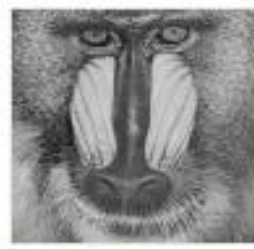

baboon

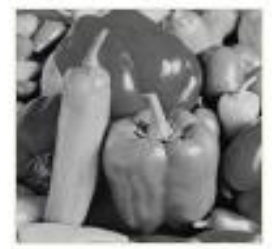

peppers

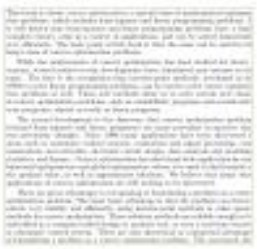

TextImage

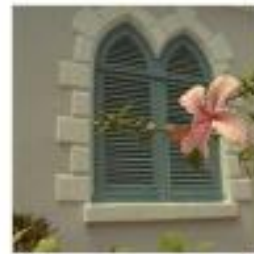

kodim07

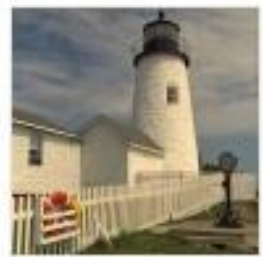

kodim19

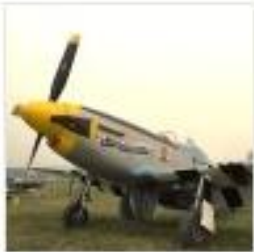

$\operatorname{kodim} 20$

Fig 3: Seven test images including four grayscale images and three color images

Table 1. Comparison of several super resolution methods in terms of PSNR

\begin{tabular}{|c|c|c|c|c|c|}
\hline Name/Method & bicubic & Yang & Zeyde & SD+AL & MD+OGMM+AL \\
\hline Lena & 30.61 & 31.05 & 31.19 & 31.54 & 31.85 \\
\hline Baboon & 21.14 & 21.27 & 20.99 & 21.14 & 21.21 \\
\hline Peppers & 31.11 & 31.62 & 32.05 & 32.32 & 32.41 \\
\hline TextImage & 14.89 & 15.13 & 14.95 & 14.99 & 15.00 \\
\hline AverageGray & 24.44 & 24.77 & 24.80 & 25.00 & $\mathbf{2 5 . 1 2}$ \\
\hline- & - & - & - & - & - \\
\hline kodim07 & 32.49 & 32.11 & 32.23 & 32.49 & 33.62 \\
\hline kodim19 & 24.72 & 24.96 & 24.25 & 24.74 & 24.89 \\
\hline kodim20 & 28.77 & 29.42 & 28.98 & 29.64 & 29.65 \\
\hline AverageY & 28.66 & 28.83 & 28.49 & 28.96 & $\mathbf{2 9 . 3 9}$ \\
\hline- & - & - & - & - & - \\
\hline AverageAll & 26.25 & 26.51 & 26.38 & 26.69 & $\mathbf{2 6 . 9 5}$ \\
\hline
\end{tabular}




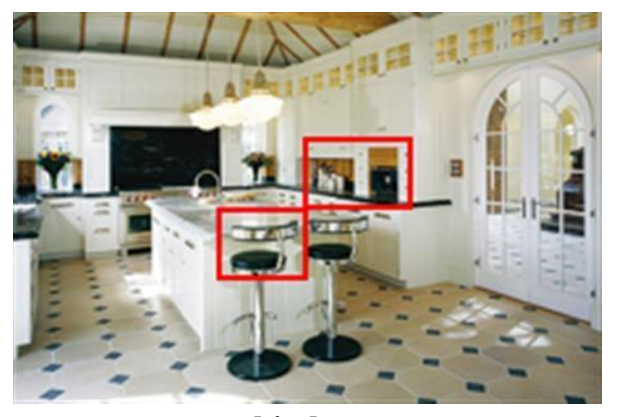

kitchen

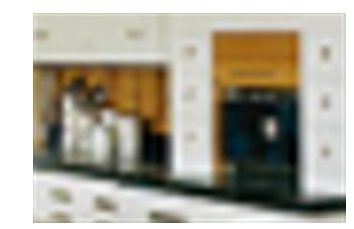

(a)

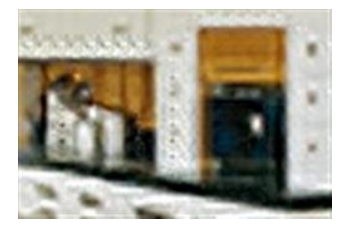

(b)

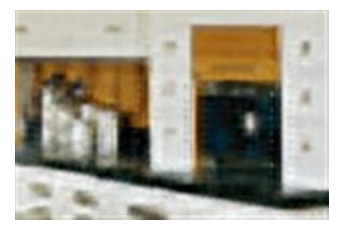

(c)

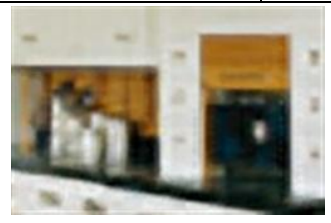

(d)

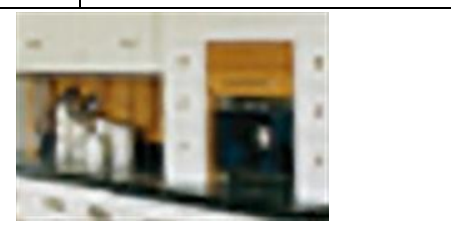

(e)

Fig 4: The results of different methods. (a) bicubic (b) Yang et al. (c) Zeyde et al. (d) SD+AL (e) MD+OGMM+AL

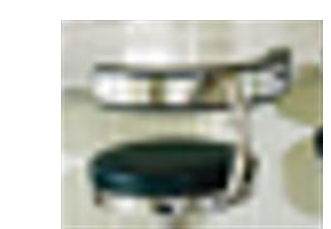

(a)

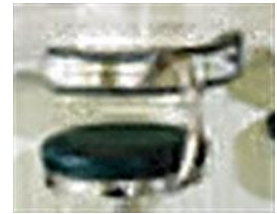

(b)

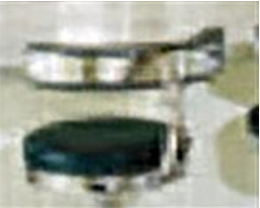

(c)

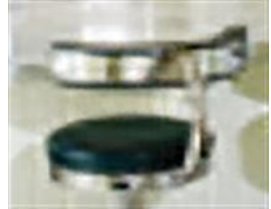

(d)

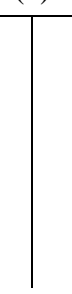

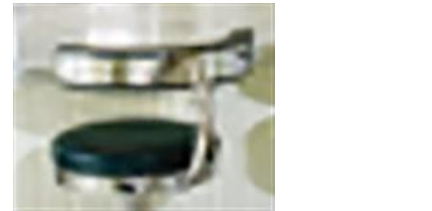

(e)

Fig 5: The results of different methods. (a) bicubic (b) Yang et al. (c) Zeyde et al. (d) SD+AL (e) MD+OGMM+AL 


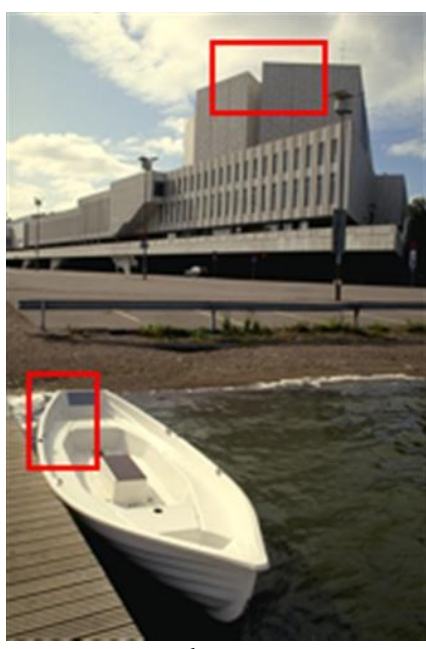

boat

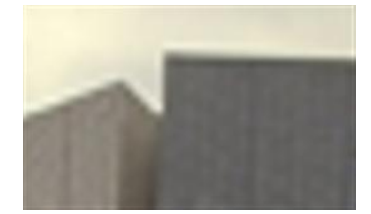

(a)

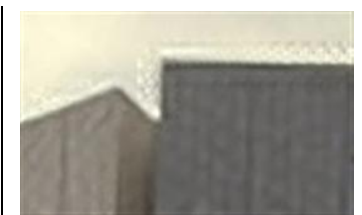

(b)
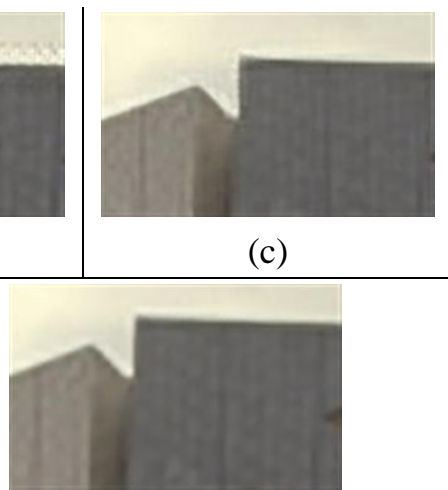

(e)

Fig 6: The results of different methods. (a) bicubic (b) Yang et al. (c) Zeyde et al. (d) SD+AL (e) MD+OGMM+AL

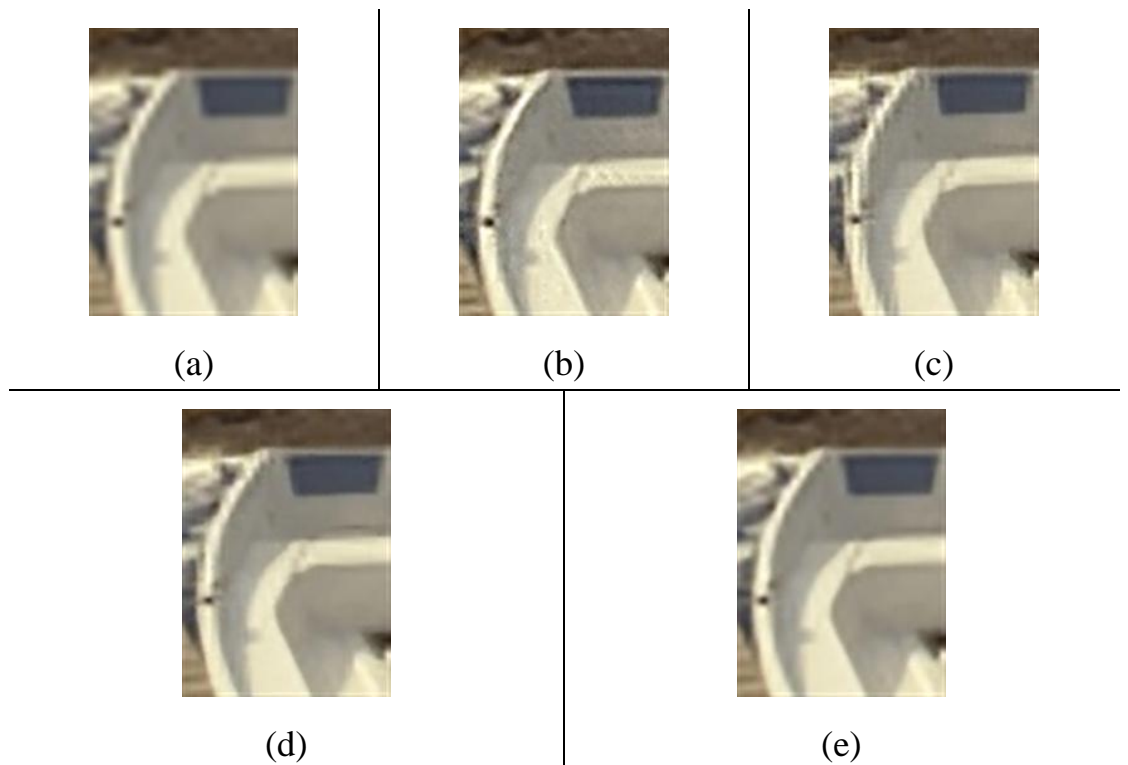

(e)

Fig 7: The results of different methods. (a) bicubic (b) Yang et al. (c) Zeyde et al. (d) SD+AL (e) MD+OGMM+AL

\section{CONCLUSIONS}

Multi-groups of coupled dictionary and its alternative learning are proposed in this paper. In learning phase, OGMM is adopted to classify image patches in principal subspace of the low resolution features. Then, joint features composed of residual features and low resolution features compressed in 
respective category space are learned by alternative learning to produce a coupled dictionary for each image patch category. In reconstruction phase, low resolution feature of each input image patch is first attempted to be reconstructed by using low resolution part of couple dictionary. Next, the dictionary that has gained least reconstruction errors is used to estimate the residual patch. Finally, the residual image and low resolution image are combined to generate a high resolution image.

Multi-redundant dictionaries scheme is also applicable to image self-learning. The reason of choosing an external image set is as follows: 1) diversify the patch types for dictionary learning and increase the quantity of learning data; 2) prevent over-fitting due to the insufficient data caused by a too small input image. It is well known that over-fitting will deteriorate the performance of dictionary.3) the dictionary learned from a specific image is only proper for itself; hence dictionary needs to be relearn for each new image. Instead, general dictionary learned from an outer image set could be used to reconstruct arbitrary image without relearn. Further research maybe the adaptive strategy: online modify the general dictionary adaptively to a specific image so as to reach a good efficiency-effectiveness tradeoff.

In fact, learning-based super-resolution reconstruction is a highly open problem. It includes image patch feature extraction, the feature compression, the atom number and learning iteration number for dictionary learning and etc. Currently, these issues are usually solved empirically and lack of theoretical analysis. In the proposed scheme, the more advanced image patch category model and reconstruction scheme are both worthy of further studying topics.

\section{REFERENCES}

[1] W. Freeman, T. Jones, E. Pasztor, "Example-based super-resolution", IEEE Computer Graphics and Applications, 22(2), pp. 56-65, 2002.

[2] H. Chang, Y. Yeung, Y. Xiong, "Super-resolution through neighbor embedding", IEEE Conference on Computer Vision and Pattern Recognition, vol. 1, pp. 275-282, 2004.

[3] T. Chan, J. Zhang, "Improved super-resolution through residual neighbor embedding", Journal of Guangxi Normal University, 24(4), pp. 211-214, 2006.
[4] T. Chan, J. Zhang, J. Pu, "Neighbor embedding based super-resolution algorithm through edge detection and feature selection", Pattern Recognition Letters, 30(5), pp. 494-502, 2009.

[5] K. Ni, T. Nguyen, "Image super-resolution using support vector regression", IEEE Transactions on Image Processing, 16(6), pp. 1596-1610, 2007.

[6] J. Yang, J. Wright, et al., "Image super-resolution as sparse representation of raw image patches", IEEE Conference on Computer Vision and Pattern Recognition, pp. $1-8,2008$

[7] J. Yang, J. Wright, T. Huang, Y. Ma, "Image super-resolution via sparse representation", IEEE Transactions on Image Processing, 19(11), pp. 2861-2873, 2010.

[8] R. Zeyde, M. Elad, M. Protter, "On single image scale-up using sparse-representations", Curves \& Surfaces, Avignon-France, June 24-30, 2010.

[9] M. Aharon, M. Elad, A. Bruckstein, "The K-SVD: An algorithm for designing of over-complete dictionaries for sparse representations", IEEE Transactions on Signal Processing, 54(11), pp. 4311-4322, 2006.

[10] K. Engan, S. Aase, "Method of optimal directions for frame design", IEEE International Conference on Acoustics, Speech and Signal Processing, vol. 5, pp. 2443-2446, 1999.

[11] M. Yang, C. Chu, Y. Wang, "Learning Sparse Image Representation with Support Vector Regression for Single-Image Super-Resolution", IEEE International Conference on Image Processing, pp. 1973-1976, 2010

[12] R. Zhang, X. Ding, "Offline handwritten numeral recognition using orthogonal Gaussian mixture model", In Proc. IEEE Int. Conf. on Image Processing, pp. 1126-1129, 2001.

[13] Zhang Xuegong, The nature of the Statistical Learning Theory, Beijing: Tsinghua University Press, 2000.

[14] G. Davis, S. Mallat, Z. Zhang, "Adaptive time-frequency decompositions", SPIE Journal of Optical Engineering, 33(7), pp. 2183-2191, 1994. 